\title{
Chiral single-wall gold nanotubes
}

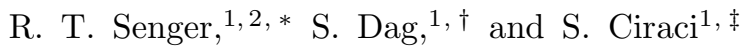 \\ ${ }^{1}$ Department of Physics, Bilkent University, 06800 Ankara, Turkey \\ ${ }^{2}$ TÜBITAK - UEKAE, P.K.74, 41470 Gebze, Kocaeli, Turkey
}

(Dated: November 15, 2018)

\begin{abstract}
Based on first-principles calculations we show that gold atoms can form both free-standing and tipsuspended chiral single-wall nanotubes composed of helical atomic strands. Free-standing, infinite $(5,5)$ tube is found to be energetically the most favorable. While energetically less favorable, the experimentally observed $(5,3)$ tube stretching between two tips corresponds to a local minimum in the string tension. Similarly, the $(4,3)$ tube is predicted as a favorable structure yet to be observed experimentally. Analysis of band structure, charge density, and quantum ballistic conductance suggests that the current on these wires is less chiral than expected, and there is no direct correlation between the numbers of conduction channels and helical strands.
\end{abstract}

PACS numbers: $61.46 .+\mathrm{w}, 73.22 . \mathrm{f}, 73.63 . \mathrm{Nm}$

Current trends in miniaturization of electronic devices have motivated a growing interest in various nanoscale structures. Of these structures nanowires constitute an important class in nanoelectronics with their potential applications as nanodevices or as connectors between them. Properties of very thin metal wires are actively studied both experimentally and theoretically $1,2,3,3,4$, 5, 6, 7, 8, 9, 10]; their formation in the form of coaxial shells having helical structures, as well as single atomic chains hanging between two electrodes have been predicted for $\mathrm{Cu}$ [5], $\mathrm{Al}$ and $\mathrm{Pb}$ [6], and $\mathrm{Au}$ [7]. Synthesis of single $\mathrm{Au}$ atom chain suspended between two Au electrodes has been a real breakthrough in nanotechnology 11, 12. Furthermore, in UHV-TEM experiments it has been shown that $\mathrm{Au}$ nanobridges can transform into several nanometers long regular chiral nanowires suspended between two $\mathrm{Au}$ electrodes. Interestingly, in agreement with previous theoretical studies, these thin nanowires have the form of helical multiwall structures of specific "magic" sizes [8].

In recent UHV-TEM experiments evidence is found for the formation of $\mathrm{Pt}$ and $\mathrm{Au}$ single-wall nanotubes (SWNT): For Pt, the tubes consist of 5 or 6 atomic rows that coil helically around the axis of the tube [13]. In the case of gold, the SWNT was observed to be composed of 5 helical strands [9]. The shell of those SWNTs can be constructed from rolling of a triangular network of gold atoms onto a cylinder of radius $R$ as described in Fig. 1 Similar to carbon nanotubes, the $(n, m)$ notation defines the structure of the tube. According to the work by Oshima et al. 9 ] the $(5,3)$ SWNT (without a linear strand at the center) was a long-lived metastable structure that has been observed between $(7,3)$ wire (with a strand at the center) and single Au atomic chain synthesized during electron beam thinning of $\mathrm{Au}$ thin foil. Apart from being the first observation of a Au chiral SWNT, this interesting result has posed several important questions as to why only $(5,3)$ tube is observed among tubes with $n=5$; what other tubes with $n<5$ are metastable.
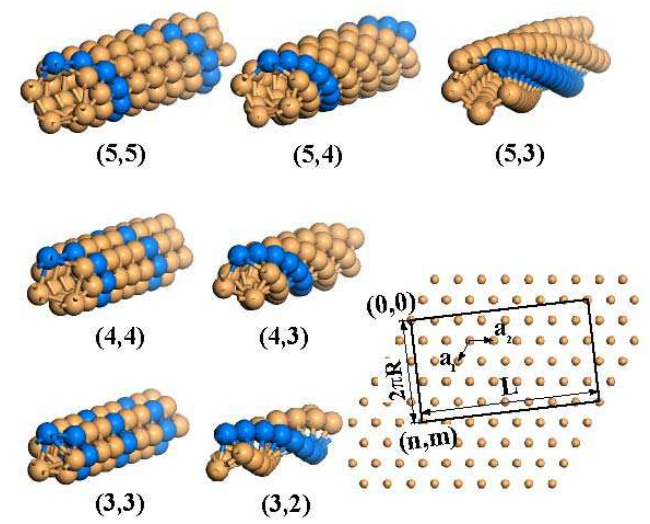

FIG. 1: Atomic structure of $(n, m)$ gold SWNTs obtained by cylindrical folding of the $2 \mathrm{D}$ triangular lattice. Basis vectors of the $2 \mathrm{D}$ lattice are $\mathbf{a}_{1}$ and $\mathbf{a}_{2}$; the chiral vector $\mathbf{C}=n \mathbf{a}_{1}+$ $m \mathbf{a}_{2}$, such that the tube circumference is $|\mathbf{C}|$, and radius $R=$ $\left(n^{2}+m^{2}-n m\right)^{1 / 2}\left|\mathbf{a}_{\mathbf{1}}\right| / \mathbf{2} \pi$. An $(n, m)$ tube has $n$ helical strands and $m$ defines the chirality. A dark helical strand of atoms highlights the chirality of the tubes. The radius $R$ and period $L$ are modified upon relaxation of the tubular structure.

While the $(5,3) \mathrm{Au}$ tube being attached to electrodes is metastable, can free-standing Au SWNTs be stable in the absence of central linear strand? How does the chirality of helical strands influence the ballistic transport? So far neither these questions have been addressed, nor the existence of gold SWNTs has been theoretically demonstrated.

In this letter, we show that tubular structures of gold can indeed exist and display interesting electronic and transport properties. Moreover, we explain why only a specific SWNT suspended between two gold tips has been observed experimentally. As single-wall tubular structures of gold, we considered all possible cases with $n=5,4,3$ and $n \geq m>n / 2$, since $(n, n-m)$ tubes are equivalent to $(n, m)$ with opposite chirality, and tubes with $n \geq 6$ have large enough radii to accommodate 
TABLE I: Structural properties and energetics of relaxed chiral gold nanotubes $(n, m)$. There are $N$ atoms in one unit cell which has length $L$ and radius $R$, both expressed in $\AA$ units. $E_{b}$ and $E_{c}$ are the binding and curvature energies per atom in units of $\mathrm{eV}$, respectively. The string tension of the tipsuspended tube, $f$, has units $\mathrm{eV} / \AA$.

\begin{tabular}{crrcccc}
\hline \hline Structure & \multicolumn{1}{c}{$N$} & \multicolumn{1}{c}{$L$} & $R$ & $E_{b}$ & $E_{c}$ & $f$ \\
\hline$(5,5)$ & 10 & 4.63 & 2.44 & 2.66 & 0.18 & 1.188 \\
$(5,4)$ & 14 & 7.15 & 2.24 & 2.60 & 0.24 & 1.179 \\
$(5,3)$ & 38 & 20.73 & 2.12 & 2.58 & 0.26 & 1.154 \\
$(4,4)$ & 8 & 4.60 & 2.04 & 2.54 & 0.30 & 1.166 \\
$(4,3)$ & 26 & 16.91 & 1.85 & 2.52 & 0.32 & 1.062 \\
$(3,3)$ & 6 & 4.39 & 1.71 & 2.41 & 0.43 & 1.083 \\
$(3,2)$ & 14 & 12.28 & 1.51 & 2.31 & 0.53 & 1.017 \\
\hline \hline
\end{tabular}

an extra linear strand of gold atoms, therefore lacking a tubular character. In particular, $(4,2)$ structure also has a non-tubular form corresponding to a dumbbell chain and is not included in our considerations. The structure of $(5,3)$ tube deduced experimentally has a period approximately five times larger than the lattice parameter of the ideal tube rolled from the undeformed gold sheet [9]. The periodicity length $L$ of the chiral tubes can be altered by a small axial shear. This is achieved by applying a shear strain of $\epsilon \| \mathbf{a}_{2}$ in the triangular network. We take $\epsilon=0$ to achieve the periodicity of the $(5,3)$ tube through $2 \pi / 5$ rotation of helical strands and hence to reduce the number of atoms in the supercell from 190 to 38. Omitting such a small strain $(\epsilon=0.005$ for $(5,3)$ tube $[9])$ does not affect our conclusions, but cuts down computational effort dramatically. We carried out total energy and electronic structure calculations on seven different tubular structures shown in Fig. 1 using first-principles pseudopotential plane-wave method [14] within generalized gradient approximation (GGA). All the atomic positions and lattice parameters of tubular structures have been optimized through lowering total energy, atomic force, and stress. The stability of relaxed structures are tested also by ab-initio molecular dynamic calculations carried out at $T=800 \mathrm{~K}$. Spin-relaxed calculations yielded zero total magnetic moments for the structures in Fig. 1 The analysis of quantum ballistic conductance has been performed by using an ab-initio transport software based on localized basis sets and non-equilibrium Green's function formalism [15]. The structural properties of the optimized gold SWNTs are summarized in Table \

The binding (or cohesive) energy $E_{b}(n, m)=E_{T}(A)-$ $E_{T}(n, m) / N$, is calculated as the difference between the energy of single $\mathrm{Au}$ atom, $E_{T}(A)$, and the total energy (per atom) of the fully relaxed $(n, m)$ tubular form having $N$ atoms in the unit cell, $E_{T}(n, m) / N$. Accordingly, $E_{b}>$ 0 (exothermic) indicates a stable structure corresponding to a local minimum on the Born-Oppenheimer surface. The curvature energy is the energy required to form a

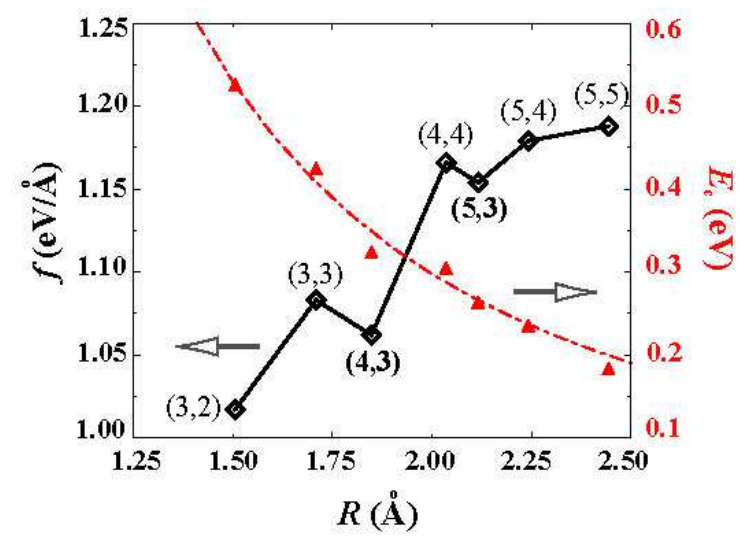

FIG. 2: Triangles are calculated curvature energy $E_{c}$ of freestanding SWNT. Dash-dotted curve is the best fit in the form $\alpha / R^{2}\left(\alpha=1.19 \mathrm{eV} \AA^{2}\right)$. Diamonds are calculated string tension $f$ of suspended $(n, m)$ gold nanotubes at zero temperature. The local minima of $f$ indicate that $(5,3)$ and $(4,3)$ SWNTs are magic.

tubular form by folding the 2D triangular network, and is expressed as $E_{c}(n, m)=E_{b}(2 \mathrm{D})-E_{b}(n, m)$, in terms of the difference of the binding energies in the closedpacked (111) atomic plane and in the $(n, m)$ tube. For the relaxed $2 \mathrm{D}$ triangular network we found $E_{b}(2 \mathrm{D})=2.84$ $\mathrm{eV}$ /atom. Normally, $E_{c}(n, m)$ increases with decreasing $R$. The calculated curvature energies comply with the expression obtained from classical elasticity theory $E_{c}=$ $Y w^{3} \Omega / 24 R^{2}$ ( $Y$ : Young's modulus, $w$ : thickness of the tube, $\Omega$ : atomic volume), and are fitted to an expression $E_{c}=\alpha / R^{2}$ in Fig. 2

All the gold nanotubes given in Table【are stable when they are standing free. The cohesive energy values, $E_{b}$, given in Table \ gradually decreases with decreasing $R$. The distribution of calculated $\mathrm{Au}-\mathrm{Au}$ bond lengths in the relaxed $(5,5)$ tube has a sharp peak at $d=2.76 \AA$, and relatively weaker individual peaks in the range of $2.85<d<2.89 \AA$. This distribution is, however, modified in the $(5,4)$ and $(5,3)$ tubes, where the sharp peak at $d=2.76 \AA$ tends to weaken and distribute in a wider range. The shortest $d$ 's correspond to the bonds forming the helical strands. As far as applications as interconnect or nanodevice are concerned, it is important to know whether the free-standing but finite length gold SWNTs are stable. The structure optimization of free $4 L$ long $(5,5)$ SWNT with open ends has resulted in a stable structure with negligible rearrangements of atoms relative to the infinite tube. Most importantly, the open ends have not been capped. The stability of gold SWNTs against clustering is somewhat nontrivial, though not totaly unexpected. Noting that free-standing finite zigzag chains of gold are found to be stable [16], the present tubular structures are expected to be even more resistant to clustering owing to their higher atomic coordination.

Among three SWNTs with $n=5$, the $(5,5)$ tube is 
energetically the most favorable. This situation is seemingly in disagreement with the experiment indicating that $(5,3)$ gold tube is the structure observed during the thinning process of gold nanowires [9]. Nevertheless, this apparent contradiction is reconciled by the fact that the calculations are performed for free-standing infinite tubes, whereas the experiment is for a finite tube stretching between two gold electrodes. Hence, $E_{b}$ should not be taken as a criterion to decide on the long-lived metastable states of suspended nanowires.

Introducing the criterion of minimum string tension rather than the total free energy for the stability of nanowires, Tosatti et al. [10] have theoretically investigated a class of gold nanowire structures having a single helical shell covering a central linear strand of atoms. They found that the wire having $(7,3)$ outer shell exhibits the minimum string tension and was specified as "magic", in agreement with observation. Here, we carry out string tension analysis for single-wall gold nanotubes without a central strand. The string tension $f$ of a nanowire is defined through the consideration of the positive work done in drawing the wire out of the tips, and is given by [10], $f=F-\mu N / L$. Here, $F$ is free energy of the one unit cell of the wire. At zero temperature, $F$ equals to the total energy $E$ of the wire; $\mu$ is the chemical potential of bulk gold, and is calculated to be $\mu \simeq-3.2 \mathrm{eV}$ within GGA, in consistence with the calculations made for the tubes. Calculated values of the string tension do not exhibit a monotonic decrease as a function of $R$ as displayed in Fig. 2 In the plot one immediately recognizes that $(5,3)$ and $(4,3)$ SWNTs have lower string tension values as compared to those of their immediate neighboring structures, thus they are favorable magic structures of gold SWNTs. Aside from the reported $(5,3)$ tube 9$]$, our analysis predicts that the $(4,3)$ tube with $R=1.85$ $\AA$ is another candidate for being a "magic" structure which is not observed yet. It appears that the $(5,3)$ gold SWNT is favored, since it lowers the tension exerted by two gold tips. In principle, the string tension calculation and the geometric relaxation of the structures should be performed self-consistently. The $f$ values reported in Table \ however, are results of the first iteration obtained by using the $F$ and $L$ values of the bare unstrained tubes. Nevertheless, we tested that the reduction in $f$ after the full self-consistent calculation is less than $0.3 \%$ for the $(5,3)$ tube, too minor to have any implications on our conclusions.

Calculated energy band structures and ab-initio ballistic conductance plots of some infinite SWNTs presented in Fig. 3] are of particular interest. The tubular character is demonstrated by contour plots of charge density, $\rho_{T}(\mathbf{r})=\sum_{i, k}^{o c c} \Psi_{i}^{*}(\mathbf{r}, k) \Psi_{i}(\mathbf{r}, k)$. Here $\rho_{T}(\mathbf{r})$ is dramatically different from that of gold nanowires with a strand at the center. Bands near the Fermi level are derived mainly from the Au-6s orbitals; one band displays significant $5 d_{z^{2}}$ hybridization. Flat $5 d$-bands occur $\sim 1 \mathrm{eV}$

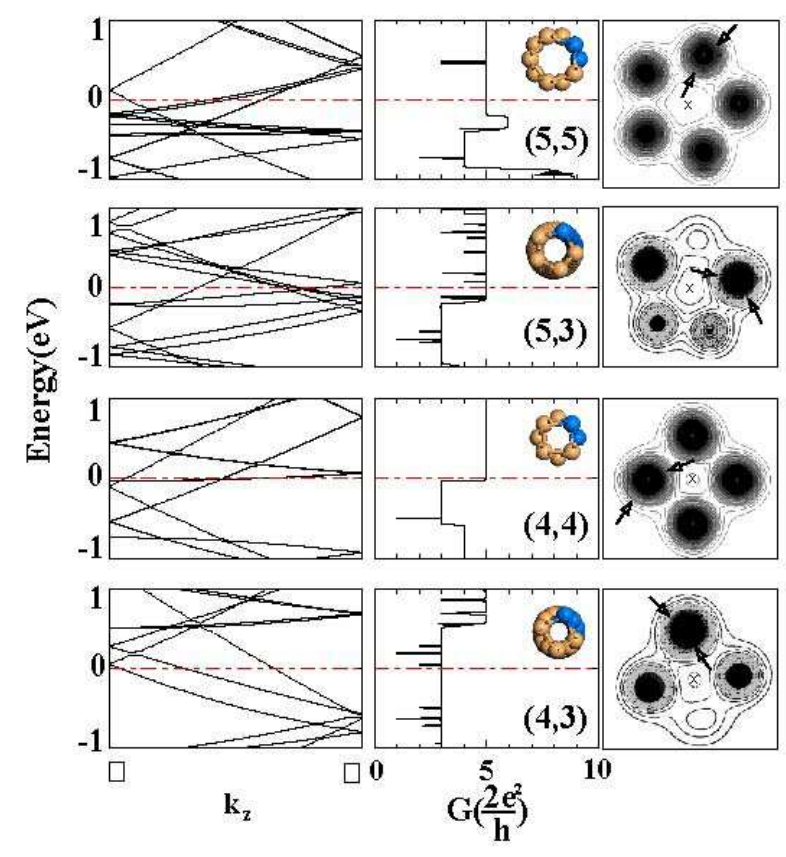

FIG. 3: Electronic energy-band structure and ballistic conductance of infinite $(5,5),(5,3),(4,4)$, and $(4,3)$ gold SWNTs. The Fermi level is set to zero in each panel. Right panels are contour plots of the total charge density on a plane perpendicular to the tube axis. The charge density is negligible at the centers of the tubes.

below the Fermi level. Despite 1D character of SWNTs, the bands which cross the Fermi level do not allow for Peierls distortion. The character of states near the Fermi level of $(5,3)$ tube is revealed by the density of states plots in Fig. 4 A single chain of gold atoms has unit quantum conductance (i.e. $G_{0}=2 e^{2} / h$ ) [11, 12]. It has been usually contemplated that one conductance channel is associated with each helical strand. Accordingly, the ballistic conductance of $n$-strand gold nanotube would be about $n G_{0}$. The three infinite SWNTs with $n=5$ have indeed equilibrium conductance values of $5 G_{0}$. However, of the 4 -strand nanotubes $(4,4)$ also has $5 G_{0}$ conductance, while the $(4,3)$ structure has only three channels for the ballistic conductance. The 3-strand family of nanotubes have $3 G_{0}$ conductance. Since the number of bands crossing the Fermi level determines the conductance of an infinite SWNT, there is no direct correlation between numbers of strands and current transporting channels, rather the cross section of the tube is expected to be crucial in determining the number of channels. Indeed, a minor reduction in the cross section area of the $(4,4)$ tube due to axial stretching reduces the number of channels from 5 to 3 . Dips of size $G_{0}$ or $2 G_{0}$ in the conductance plots are another interesting feature one notes. They are due to small gap openings in the energy band diagrams of these helical structures. A recent calculation on the conductance of helical nanowires attributes such 


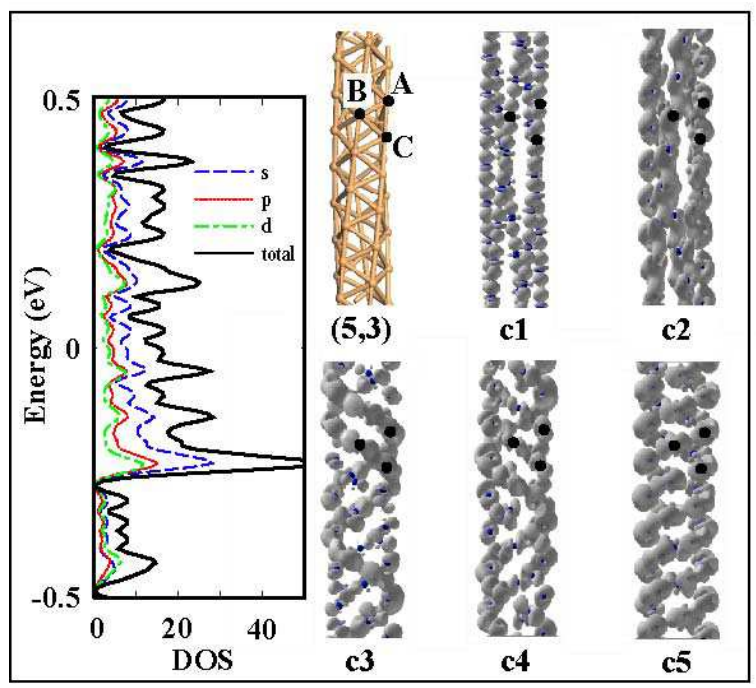

FIG. 4: Left: Total and orbital-decomposed densities of states around the Fermi level $(E=0)$ of $(5,3)$ gold SWNT. Right: A schematic description of the structure, and isosurfaces for the charge densities of the five states at the Fermi level corresponding to current transporting channels $\mathrm{c} 1, \mathrm{c} 2, \ldots \mathrm{c} 5$. Three reference points $\mathrm{A}, \mathrm{B}, \mathrm{C}$ are shown by dark spots on each plot.

characteristic dips to the non-circular cross-section of the wires 17. Note that the conductance plots of $(5,3)$ and $(4,3)$ tubes in Fig. 3 have more of those dips since they are more chiral. Finally, we calculated the conductance of finite size (one unit cell) $(5,3)$ tube which is connected to two fcc gold electrodes through single Au atoms from both ends, and found $G=1.75 G_{0}$. Dramatic reduction from $5 G_{0}$ (the conductance of infinite tube) is attributed to the contacts with electrodes.

Prediction of chiral currents in chiral (also mechanically stretched) single-wall carbon nanotubes has been of interest because of the self-inductance and nanocoil effects [18]. In principle, chiral current passing through a one-micron $(5,3)$ SWNT can induce magnetic fields of several Tesla [19]. According to Altshuler-Aronov-Spivak effect [20] electrons circling a cylindrical conductor that encloses a magnetic flux $\Phi$ give rise to a periodic oscillating resistance as a function of $\Phi$. The observed shortperiod oscillations in the magnetoresistance of multi-wall carbon nanotubes have been attributed to chiral currents developed by mechanical stretching of them 21. It is of interest to reveal whether the electrical current passing through a $(5,3)$ SWNT has chirality due to the helical

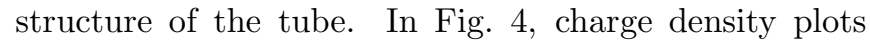
of five states at the Fermi level, corresponding to the conduction channels, clearly indicates the chirality. The geometry of the atomic positions in the $(5,3)$ tube provides three distinct circumferential directions for the flow of the current. These directions can be defined as $\mathrm{AB}$, $\mathrm{AC}$, and $\mathrm{BC}$ in terms of the reference points $\mathrm{A}, \mathrm{B}, \mathrm{C}$

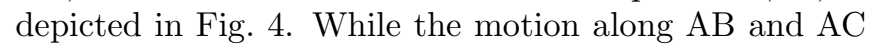

directions corresponds to right-handed helices, the helical path along the BC direction is left-handed. The period and direction of chirality are different for different channels; $\mathrm{c} 3$ and c4 have opposite chiral directions compared to $\mathrm{c} 1, \mathrm{c} 2$, and $\mathrm{c} 5$. Hence, the resultant chirality effect may be weaker than one expects. For the infinite tube, being a transmission eigenchannel of the system, each channel contributes a unit quantum conductance. However, for suspended tubes, the chirality of the net current depends on the combined "nanocoil" effects of the conduction channels, as well as on the contacts.

In conclusion, we showed that free-standing gold chiral $(n, m)$ tubes with $3 \leq n \leq 5$ are stable and exhibit novel electronic and transport properties. Our analysis explains why the experimentally observed $(5,3)$ tube suspended between two gold tips is favored, and indicates that the string-tension criterion introduced by Tosatti et al [10] is also valid for tubular structures. Using this criterion we predict that tip-suspended $(4,3)$ chiral gold tube is another structure that can be observed. We found that there is no direct correlation between the numbers of conduction channels and helical strands making the tubular structure. Current transporting states display different periods and chirality, the combined effects of which lead to weaker chiral currents on SWNTs.

* Electronic address: senger@fen.bilkent.edu.tr

$\dagger$ Electronic address: sefa@fen.bilkent.edu.tr

¥ Electronic address: ciraci@fen.bilkent.edu.tr

[1] J. K. Gimzewski and R. Möller, Phys. Rev. B 36, 1284 (1987).

[2] S. Ciraci and E. Tekman, Phys. Rev B 40, 11969 (1989).

[3] N. Agraït et al., Phys. Rev. B 47, 12345 (1993); N. Agraït et al., Phys. Rev. Lett. 74, 3995 (1995).

[4] J. I. Pascual et al., Phys. Rev. Lett. 711852 (1993).

[5] H. Mehrez and S. Ciraci, Phys. Rev. B 56, 12632 (1997).

[6] O. Gülseren et al., Phys. Rev. Lett. 80, 3775 (1998).

[7] M. R. Sorensen et al., Phys. Rev. B 57, 3283 (1998).

[8] Y. Kondo and K. Takayanagi, Science 289, 606 (2000).

[9] Y. Oshima et al., Phys. Rev. Lett. 91, 205503 (2003).

[10] E. Tosatti et al., Science 291, 288 (2001).

[11] H. Ohnishi et al., Nature (London) 395, 780 (1998).

[12] A. I. Yanson et al., Nature (London) 395, 783 (1998).

[13] Y. Oshima et al., Phys. Rev. B 65, 121401 (2002).

[14] Calculations have been performed by using the VASP software: G. Kresse and J. Hafner, Phys. Rev. B 47, 558 (1993); G. Kresse and J. Furthmüller, ibid 54, 11169 (1996).

[15] The methodology of the TRANSIESTA-C software is based on: M. Brandbyge et al., Phys. Rev. B 65, 165401 (2002). The software is provided by Atomistix Corp.

[16] D. Sánchez-Portal et al., Phys. Rev. Lett. 83, 3884 (1999).

[17] M. Okamoto et al., Phys. Rev. B 64, 033303 (2001).

[18] Y. Miyamoto et al., Phys. Rev. B 60, 13885 (1999).

[19] V. M. K. Bagci et al., Phys. Rev. B 66, 045409 (2002).

[20] B. L. Altshuler et al., JETP Lett. 33, 94 (1981). 
[21] A. Bachtold et al., Nature (London) 397, 673 (1999). 